\title{
Chronic Ankle Instability Does Not Influence Tibiofemoral Contact Forces during Drop Landings ${ }^{\dagger}$
}

\author{
Yumeng Li ${ }^{1, *}$, He Wang ${ }^{2}$ and Kathy J. Simpson ${ }^{3}$ \\ 1 Department of Health and Human Performance, Texas State University, San Marcos, TX 78666, USA \\ 2 School of Kinesiology, Ball State University, Muncie, IN 47306, USA; hwang2@bsu.edu \\ 3 Department of Kinesiology, University of Georgia, Athens, GA 30602, USA; kjsimpsonuga@gmail.com \\ * Correspondence: yumeng.li@txstate.edu; Tel.: +1-706-255-2499 \\ + Presented at the 13th Conference of the International Sports Engineering Association, Online, \\ 22-26 June 2020.
}

Published: 15 June 2020

\begin{abstract}
Chronic ankle instability (CAI) is a very common sequela after ankle sprains. Previous studies observed some knee biomechanical and neuromuscular alterations of CAI that could potentially relate to the knee injury mechanism during landings. However, to our knowledge, no studies have assessed the tibiofemoral contact forces for individuals with CAI. The purpose of the study was to compare the tibiofemoral contact forces of participants with CAI versus controls during landings using a computer-simulated musculoskeletal model. Twenty-one female participants with CAI and 21 pair-matched controls performed a drop landing task on a tilted force plate. A seven-camera motion capture system and two force plates were used to test participants' lower extremity biomechanics. A musculoskeletal model was used to calculate the tibiofemoral contact forces (femur on tibia). No significant between-group differences were observed for the peak tibiofemoral contact forces $(p=0.25-0.48)$ during the landing phase based on paired $t$-tests. The group differences ranged from 0.05 to 0.58 body weight (BW). Most participants demonstrated a posterior force (peak $=\sim 1.1 \mathrm{BW}$ ) for most of the landing phase and a medial force (peak $=\sim 0.9 \mathrm{BW}$ ) and a large compressive force (peak $=\sim 10 \mathrm{BW}$ ) in the landing phase. We conclude that CAI may not be related to the increased tibiofemoral contract forces or knee injury mechanisms during landings on tilted surfaces.
\end{abstract}

Keywords: ankle sprain; computer simulation; musculoskeletal model; impact injuries

\section{Introduction}

Chronic ankle instability (CAI) is a very common sequela after ankle sprains [1]. Previous studies also investigated the influence of ankle sprains on knee injury mechanisms. Two studies suggested that having had a prior ankle sprain may be related to knee injury (e.g., anterior cruciate ligament injury) based on a significant association between ankle sprain history and knee injury history $[2,3]$. Other studies observed some knee biomechanical alterations of CAI that could potentially relate to the knee injury mechanism during landings. These alterations of CAI, compared with controls, included reduced knee flexion angles [4], greater knee extension and internal rotation moments [5], and higher quadriceps to hamstrings activation ratio [6].

However, the effect of CAI on knee injury mechanism is not well understood without estimating the tibiofemoral contract forces. Therefore, the purpose of the study was to compare the tibiofemoral contract forces of participants with CAI versus controls during landings using a musculoskeletal model. We hypothesized that the CAI group would display greater tibiofemoral contract forces compared to controls. 


\section{Method}

\subsection{Participants}

Twenty-one female participants with CAI were recruited based on previously published guidelines and questionnaires [7]; 21 healthy controls were recruited to pair-match with the CAI participants on gender, age $( \pm 3$ years), height $( \pm 2.5 \mathrm{~cm})$, body mass $( \pm 4.5 \mathrm{~kg})$ and physical activity level ( $\pm 2 \mathrm{~h} / \mathrm{wk}$ in moderate and vigorous activities) (Table 1$)$. All participants were physically active and had experience in landing-related sports.

Table 1. Participants' characteristics.

\begin{tabular}{ccc}
\hline Variable & CAI & Controls \\
\hline Sample Size & 21 & 21 \\
Body mass $(\mathrm{kg})$ & $64.4 \pm 12.4$ & $64.4 \pm 11.9$ \\
Height $(\mathrm{cm})$ & $164 \pm 6$ & $165 \pm 6$ \\
Age (years) & $21 \pm 2$ & $21 \pm 2$ \\
CAIT score & $19.3 \pm 6.0$ & $29.5 \pm 0.9$ \\
IdFAI score & $22.2 \pm 9.2$ & $1.3 \pm 2.1$ \\
\hline
\end{tabular}

Note: CAIT $=$ Cumberland ankle instability tool; IdFAI = Identification of functional ankle instability.

\subsection{Test Protocol}

Twenty-nine reflective markers were placed on the trunk, pelvis, and lower extremities, and locations were collected by a 7-camera 3-D motion capture system. The ground reaction forces (GRF) were measured by two force plates at $2040 \mathrm{~Hz}$. Participants stood on a 30-cm high box and stepped forward with the CAI limb followed by the other limb and landed with the CAI limb on a 25-degree tilted force plate and the other foot on the flat force plate (Figure 1). For controls, the test limb was the limb that was pair-matched with limb dominancy of the CAI participants. Ten landing trials were collected and analyzed.

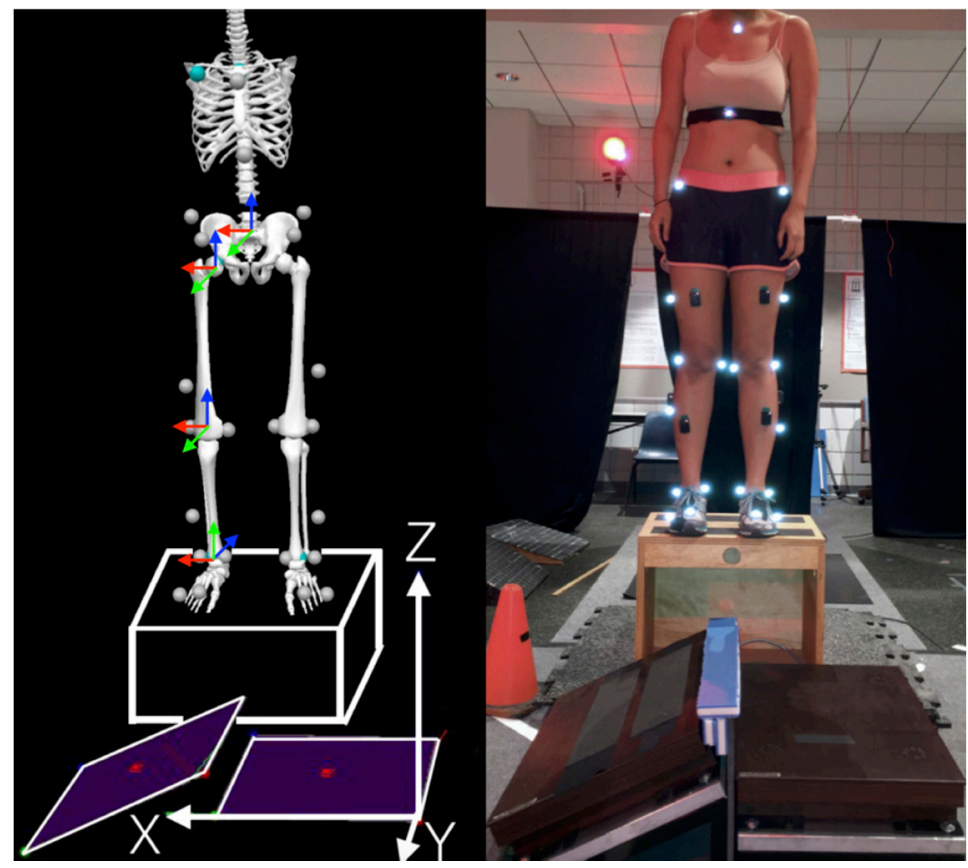

Figure 1. Experimental setup for the drop landing test.

\subsection{Data Analysis}

3-D marker coordinates were filtered with a low-pass filter at $15 \mathrm{~Hz}$. Participants' lowerextremity musculoskeletal models including generic geometrics of pelvis, femur, tibia, and foot were 
created in LifeMOD 2012 (LifeModeler Inc., San Clemente, CA, USA), a plug-in program in ADAMS 2012 (MSC Software Corp., Newport Beach, CA, USA) that is widely utilized in musculoskeletal simulations for various human body motions. The generic lower-extremity model was then scaled based on participant mass, height, gender, and age, as well as the relative positions of the ankle, knee, and hip joints determined from the kinematic data. Tri-axis hinges, combined with passive torsional spring-dampers $\left(1 \mathrm{Nmm} /{ }^{\circ}\right.$ and $\left.0.1 \mathrm{Nmm} \cdot \mathrm{s} /{ }^{\circ}\right)$, were employed to model the joints. A total of 90 muscles were assigned to the legs. A proportional-integral-derivative feedback controller was implemented to calculate each muscle force magnitude using the error signal between the current muscle length in the forward dynamics and the recorded muscle length during the inverse dynamics simulation. The tibiofemoral contract forces (forces from the femur applied onto the proximal tibia) were computed from the difference between the resultant joint forces and the muscle forces at the knee joint. The tibiofemoral contract forces were normalized to participant body weight (BW). The peak tibiofemoral contract forces were generated and averaged across ten trials and compared between groups (CAI and controls) using paired $t$-tests $(p<0.05)$.

\section{Results}

No significant between-group differences were observed for the peak tibiofemoral contract forces ( $p=0.25-0.48$ ) during the landing phase. The group differences ranged from 0.05 to $0.58 \mathrm{BW}$. In general, for most of the participants, a posterior tibiofemoral contact force exhibited throughout most of the landing phase. In addition, both groups demonstrated a medial force (peak $=\sim 0.9 \mathrm{BW}$ ) and a large compressive force (peak $=\sim 10 \mathrm{BW}$ ) on the proximal tibia. The descriptive and group comparison outcomes are presented in Table 2.

Table 2. Descriptives (mean \pm SD) and between-group comparison outcomes of the peak tibiofemoral contract forces ( $\times$ body weight) during the landing phase.

\begin{tabular}{ccccc}
\hline Variables $(\times \mathbf{B W})$ & CAI & CON & $p$ Values & Cohen's $d$ \\
\hline Posterior Force & $1.23 \pm 0.51$ & $1.04 \pm 0.40$ & 0.14 & 0.41 \\
Medial Force & $0.88 \pm 0.19$ & $0.93 \pm 0.34$ & 0.48 & 0.18 \\
Compressive Force & $10.23 \pm 2.09$ & $9.65 \pm 1.39$ & 0.28 & 0.33 \\
\hline
\end{tabular}

\section{Discussion}

The anterior-posterior tibiofemoral force was primarily taken up by the ligaments because the friction between the tibia and femur was assumed to be negligible [8]. As the anterior cruciate ligament (ACL) is the primary restraint to anterior translation of the tibia, the posterior tibiofemoral force could correspond to ACL loading. In other words, the posterior tibiofemoral force on the tibia was majorly taken up by the ACL. In the present study, a posterior tibiofemoral force was exhibited throughout most of the landing phase for most of the participants. No group-difference in peak values was found, which could indicate that the CAI group likely experienced similar peak ACL loadings versus controls. Previous studies $[5,6]$ have observed greater knee extension and internal rotation moment, greater rectus femoris activation, but less biceps femoris activation, and suggest these altered knee biomechanics and muscle activations of CAI could relate to the increased ACL loading. However, this may not be true based on our tibiofemoral contact force data. The possible reason was that the CAI group also exhibited a greater knee flexion angle [5] that could decrease the tibial anterior shear force by decreasing the patella tendon-tibia shaft angle $[9,10]$. Therefore, it is still unclear whether CAI could increase the ACL loading during landings. Future research is warranted to estimate the ACL loading for individuals with CAI using more detailed models. The posterior tibiofemoral contract forces were also reported in previous studies [11,12]. However, possibly because landing on a tilted surface was a more demanding situation [13], the posterior tibiofemoral contract forces observed in the present study $(\mathrm{CAI}=1.23 \mathrm{BW}$; controls $=1.04 \mathrm{BW})$ was greater than that reported in flat-surface drop landings (Kernozek and Ragan, 2008: 0.15 BW; Laughlin et al., 2011: $0.6 \mathrm{BW})$. This supposition could be partly supported by the peak posterior tibiofemoral contract forces of the non-test limbs landed on the flat surface in the present study $(\sim 0.61 \mathrm{BW}$ for all 
participants, lower than that landed on the tilted surface). The higher tibiofemoral contract forces observed in the present study were possibly due to greater muscle co-contraction and also found in the compressive direction (described below).

The CAI and control groups exhibited comparable peak compressive tibiofemoral contract forces during landings. Great compressive forces are highly significant in knee injuries or diseases (e.g., knee osteoarthritis) [14]. In addition, the compressive loading with a posterior tibial plateau slope has been considered as an important mechanism of ACL injury $[15,16]$. In previous cadaver studies, a small knee flexion angle of $15^{\circ}-30^{\circ}$ with a compressive knee force of $5.4-10.8 \mathrm{KN}$ could damage the ACL $[16,17]$. The average peak compressive force was $6.2 \mathrm{KN}$ during landings in the present study; the ACL seemed vulnerable to this high force. However, unlike the cadaver studies, our participants may have exhibited knee joint muscle co-contractions (e.g., quadriceps and hamstring) during landings to stabilize the joint and resulted in a relatively large compressive force. The peak compressive forces observed in the present study $(\sim 10 \mathrm{BW})$ were greater in comparison with those reported in jump landings and sidestep cuttings (7.5-8.2 BW) [18,19]. The magnitudes were also greater than those found in sports activities ( $\sim \mathrm{BW}$ in jogging, golf, and tennis) [20] because of the higher segmental decelerations, impact forces, and muscle contractions during landings.

There were several limitations to the present study. As typical in landing studies, the skin movement artifact could influence the calculations of kinematic and kinetic variables. For a limited number of frames when the knee flexion angle was large, a higher upper bound for the muscle forces was used to find a solution. Moreover, different shoes worn by the participants could affect the landing biomechanics differently.

\section{Conclusions}

In conclusion, the CAI group demonstrated similar tibiofemoral contract forces compared with controls during drop landings on the tilted surface. We did not find any direct evidence to support that CAI could relate to knee injury mechanisms. However, to have a better understanding of the effect of CAI on knee injury in sports, tibiofemoral contract forces or ACL loadings should also be assessed in other experimental tasks (e.g., cutting maneuver, jump landings, or single-leg landings).

Funding: No funding has been received to support the present study.

Conflicts of Interest: The authors declare no conflict of interest.

\section{References}

1. Kobayashi, T.; Gamada, K. Lateral ankle sprain and chronic ankle instability: A critical review. Foot Ankle Spec. 2014, 7, 298-326.

2. Söderman, K.; Alfredson, H.; Pietilä, T.; Werner, S. Risk factors for leg injuries in female soccer players: A prospective investigation during one out-door season. Knee Surg. Sports Traumatol. Arthrosc. 2001, 9, 313-321.

3. Kramer, L.C.; Denegar, C.R.; Buckley, W.E.; Hertel, J. Factors associated with anterior cruciate ligament injury: History in female athletes. J. Sports Med. Phys. Fit. 2007, 47, 446-454.

4. Terada, M.; Pietrosimone, B.; Gribble, P.A. Individuals with chronic ankle instability exhibit altered landing knee kinematics: Potential link with the mechanism of loading for the anterior cruciate ligament. Clin. Biomech. 2014, 29, 1125-1130.

5. Li, Y.; Ko, J.; Walker, M.; Brown, C.; Schmidt, J.; Kim, S.H.; Simpson, K. Does chronic ankle instability influence knee biomechanics of females during inverted surface landings? Int. J. Sports Med. 2018, 39, 10091017.

6. Li, Y.; Ko, J.; Walker, M.A.; Brown, C.N.; Schmidt, J.D.; Kim, S.H.; Simpson, K.J. Does chronic ankle instability influence lower extremity muscle activation of females during landing? J. Electromyogr. Kinesiol. 2018, 38, 81-87.

7. Gribble, P.A.; Delahunt, E.; Bleakley, C.; Caulfield, B.; Docherty, C.L.; Fourchet, F.; Fong, D.; Hertel, J.; Hiller, C.; Kaminski, T.W.; et al. Selection criteria for patients with chronic ankle instability in controlled research: A position statement of the international ankle consortium. J. Orthop. Sports Phys. Ther. 2013, 43, $585-591$. 
8. Shin, C.S.; Chaudhari, A.M.; Andriacchi, T.P. The influence of deceleration forces on ACL strain during single-leg landing: A simulation study. J. Biomech. 2007, 40, 1145-1152.

9. Yu, B.; Garrett, W.E. Mechanisms of non-contact ACL injuries. Br. J. Sports Med. 2007, 41 (Suppl. 1), i47-i51.

10. Nunley, R.; Wright, D.; Renner, J.; Yu, B.; Garrett, W.E. Gender comparison of patellar tendon tibial shaft angle with weight bearing. Res. Sports Med. 2003, 11, 173-185.

11. Laughlin, W.A.; Weinhandl, J.T.; Kernozek, T.W.; Cobb, S.C.; Keenan, K.G.; O'Connor, K.M. The effects of single-leg landing technique on ACL loading. J. Biomech. 2011, 44, 1845-1851.

12. Kernozek, T.W.; Ragan, R.J. Estimation of anterior cruciate ligament tension from inverse dynamics data and electromyography in females during drop landing. Clin. Biomech. 2008, 23, 1279-1286.

13. Chen, Q.; Wortley, M.; Bhaskaran, D.; Milner, C.E.; Zhang, S. Is the inverted surface landing more suitable in evaluating ankle braces and ankle inversion perturbation? Clin. J. Sports Med. 2012, 22, 214-220.

14. D'Lima, D.D.; Fregly, B.J.; Patil, S.; Steklov, N.; Colwell, C.W., Jr. Knee joint forces: Prediction, measurement, and significance. Proc. Inst. Mech. Eng. Part H 2012, 226, 95-102.

15. Boden, B.P.; Torg, J.S.; Knowles, S.B.; Hewett, T.E. Video analysis of anterior cruciate ligament injury: Abnormalities in hip and ankle kinematics. Am. J. Sports Med. 2009, 37, 252-259.

16. Wall, S.J.; Rose, D.M.; Sutter, E.G.; Belkoff, S.M.; Boden, B.P. The role of axial compressive and quadriceps forces in noncontact anterior cruciate ligament injury: A cadaveric study. Am. J. Sports Med. 2012, 40, 568573.

17. Meyer, E.G.; Haut, R.C. Anterior cruciate ligament injury induced by internal tibial torsion or tibiofemoral compression. J. Biomech. 2008, 41, 3377-3383.

18. Weinhandl, J.T.; Earl-Boehm, J.E.; Ebersole, K.T.; Huddleston, W.E.; Armstrong, B.S.R.; O'Connor, K.M. Reduced hamstring strength increases anterior cruciate ligament loading during anticipated sidestep cutting. Clin. Biomech. 2014, 29, 752-759.

19. Cleather, D.J.; Goodwin, J.E.; Bull, A.M.J. Hip and knee joint loading during vertical jumping and push jerking. Clin. Biomech. 2013, 28, 98-103.

20. D'Lima, D.D.; Steklov, N.; Patil, S.; Colwell, C.W. The mark coventry award: In vivo knee forces during recreation and exercise after knee arthroplasty. Clin. Orthop. Relat. Res. 2008, 466, 2605-2611. 